\title{
Biologically Based Practices
}

National Cancer Institute

\section{Source}

National Cancer Institute. Biologically Based Practices. NCI Thesaurus. Code C61370.

A major category of Complementary and Alternative Medicine (CAM), dealing with natural and biologically-based nutritional interventions and practices. These include, but is not limited to, botanicals, animal-derived extracts, vitamins, minerals, fatty acids, amino acids, proteins, prebiotics and probiotics (live bacteria (and sometimes yeasts) found in foods such as yogurt or in dietary supplements), whole diets, and functional foods. 